\title{
Mon stress
}

\section{Anna Sax}

Lic. oec. publ., MHA, membre de la rédaction

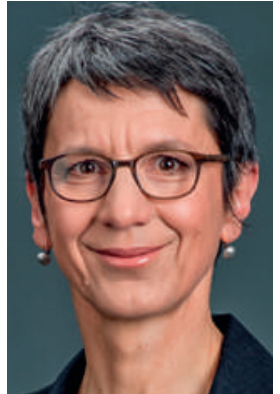

La nuit, à trois heures du matin, c'est comme une onde de choc qui me traverse tout le corps: demain, clôture de rédaction! Je suis alors gagnée par une sorte de désespoir, à la pensée du cours à préparer, des questions auxquelles il faut répondre et des offres à envoyer. Magazines et ouvrages non lus s'accumulent, je perds lentement le contrôle de la comptabilité. Pour le moment, je n'ai plus aucune idée des finances. Bien sûr, c'est le moment que choisit Internet pour être en panne, sans parler du téléphone qui fonctionne mal depuis le déménagement. Me voici complètement éveillée, essayant de mettre de l'ordre dans mes pensées. En vain. Dois-je me lever au beau milieu de la nuit pour pointer mes comptes? Non. Ca va trop loin maintenant. Ou plutôt si, je me lève et dresse une liste des choses à faire avant de retourner sous la couette. Au moins, les tâches en suspens sont maintenant fixées sur une feuille et ne polluent plus mon cerveau, qui voudrait se reposer pendant quelques heures. On connaît tous l'inutilité des ruminations nocturnes qui n'apportent rien de bien utile, une fois considérées à la lumière du jour. Je me rendors donc.

\section{Me voici complètement éveillée, essayant de mettre de l'ordre dans mes pensées.}

Je subis ces coups de stress de temps en temps depuis que je travaille, plus que jamais depuis que je suis indépendante, soit plusieurs dizaines d'années maintenant. Je me suis habituée à avoir une charge de travail variable et à ne pas toujours réussir à gérer le temps. Parfois, les e-mails disparaissent, un article est terminé trop tard ou un cours n'est pas préparé en détail, ce qui panique la perfectionniste que je suis. Heureusement, il y a deux personnes en moi. L'autre dit: Tant pis! Mettons nos baskets et allons courir! Et poursuit: Comment y arriver si on ne remarque même plus que le printemps est arrivé? A qui cela servira-t-il de propager la mauvaise humeur, sous prétexte que je ne maîtrise pas les délais? Elle susurre même avec impertinence: Personne ne remarquera que la chargée de cours que tu es improvise. Tu as assez de métier, tu trouveras toujours quelque chose.

Jusqu'à maintenant, je ne me suis pas beaucoup préoccupée des stratégies de gestion du stress et du temps, des techniques de travail efficaces et autres questions du même acabit. Je n'ai encore jamais mis en matrice l'urgent et l'important, ni établi de listes de choses à faire ou de planning hebdomadaire. Pas le temps. Avec l'expérience, j'ai découvert que les listes aidaient certes à calmer le tumulte des pensées nocturnes, mais d'ici à

\section{Quand je pense au stress, je comprends que ma} situation est privilégiée.

ce que je les respecte le lendemain... Le jour d'après surgira probablement autre chose, que je devrai régler rapidement avant même de m'attaquer à ma fameuse liste. Je me plonge donc dans un article, qui ne m'est pour l'instant d'aucune utilité, mais qui est intéressant. Qui sait, peut-être pourrai-je l'exploiter plus tard. Entretemps il est midi, et la journée a filé, car j'ai une réunion l'après-midi. Je m'y préparerai dans le train. La liste reste sur le bureau. Demain est un autre jour.

Quand je pense au stress, je comprends que ma situation est privilégiée. En effet, mon stress ne m'est pas imposé de l'extérieur. Il m'appartient, à moi seule. Aucune cheffe n'exige de moi que je fournisse plus que ce dont je suis capable. Aucun businessplan ne me contraint à vendre à d'autres personnes des choses dont elles ne veulent pas. Je ne suis pas en concurrence avec mes collègues pour faire carrière. Aucun chef n'est vexé parce que je ne ris pas à ses blagues. Quand j'y réfléchis bien, mon stress a même quelque chose de vivifiant et de stimulant. Quand je n'ai pas de rendezvous en attente, je traîne souvent sans entrain au bureau, avec une productivité proche de zéro. Mais quand c'est la panique, que la messagerie explose et que le corps réclame l'air frais du printemps... là, je me sens vraiment vivante. 